\title{
Analysis of variance with Fourier analysis of coherent data
}

\author{
PHILLIP L. EMERSON \\ Cleveland State University, Cleveland, Ohio 44115
}

\begin{abstract}
Like orthogonal polynomials, the sine and cosine components used in Fourier analysis are orthogonal under summation over equally spaced points. Therefore, they can be used in the same way as orthogonal polynomials are used with analysis of variance. They are usually used on records consisting of sequences of observations taken equally spaced in time. Analysis of variance can be performed on replicated records. The most straightforward cases occur under the following conditions: (1) The phenomenon is periodic, (2) the phenomenon is coherent (synchronized) over the various replicates, (3) the record length is an integral multiple of the fundamental period, and (4) the phenomenon is not buried too deeply in noise. Two numerical examples are examined that satisfy these conditions. Planning is illustrated for a case that violates Conditions 3 and 4.
\end{abstract}

Among behavioral researchers, it is a pleasure to discover that some new statistical or methodological problem can be understood with slight variations on concepts that are already familiar. The first advantage is that logical or mathematical derivations are much reduced. The second is one of communication. The situation is like that when two strangers discover that they share many common experiences or speak the same language. Kaplan (1983) showed us, just as Socrates showed Meno, ${ }^{1}$ that we already know a lot about Fourier analysis. We need only be made aware of our knowledge. Practically, his approach enables us to avoid much tedious discussion of details. In the present discussion, there is reference to least-squares estimation, multiple regression, stepwise polynomial regression, orthogonal polynomials, repeated-measures designs, and the assumptions underlying analysis of variance (ANOVA). Great expertise on these topics is not required. It is sufficient that these phrases be somewhat familiar and that each of us individually have an idea of where to find out more about them, if the need should arise. This discussion does not cover all aspects of statistical inference with Fourier analysis that might be of interest in psychology. Indeed, new developments are still occurring (e.g., Porges, Bohrer, Cheung, Drasgow, McCabe, \& Gideon, 1980). Instead, attention is focused on a class of experimental designs that has been chosen because it provides an easy entry into the general methodology.

\section{RESTRICTIONS AND PRECAUTIONS}

The methods discussed here are restricted in several ways. First, they assume that repeated measurements are made on subjects over time, at equally spaced intervals. Second, they assume that the error components are independent and normally distributed with zero mean and common variance, as in most other applications of
ANOVA. Most restrictive, perhaps, is the assumption of coherence of the phenomenon under study. Practically, this means time locking of some sort so that the systematic components of the response pattern are pretty much synchronized in the records from the different subjects or replicates. This rules out such things as free running EEG, but it encompasses such things as cortical potentials driven by a periodic stimulus, diurnal rhythms, and such. Note that the general assumptions of ANOVA imply noncoherence of the random component.

The word "coherence" has been applied to the idea of synchronization in physical and engineering contexts. The difference between lasers and ordinary light is that lasers are coherent, whereas ordinary light is noncoherent. An army infantry practice is often mentioned to illustrate some physical effects of coherence. It is said that troops are ordered to march route step when crossing a long bridge. The synchronized effects of a large number of men marching in step has been known to set up such vibrations as to cause a bridge to collapse. Such a principle also applies in a statistical sense. More information is obtainable from a set of synchronized data records than from the same set when the data have been shifted randomly with respect to each other. The coherent design requires time locking within conditions, but it can answer questions about time differences between conditions. The noncoherent design does not require the former and cannot answer the latter.

The methods to be described here are directly applicable to equally spaced raw data under some circumstances. If (1) the phenomenon under study is known to be periodic and relatively smooth, (2) the record length is an integral multiple of the fundamental period, and (3) the true nonrandom components are not buried too deeply in noise, then the methods will give meaningful results in most cases. If some of these conditions do not hold, and if one is attempting to estimate the 
true frequency components of continuous patterns, then the direct application of the procedures described here could turn out to be misleading. Blackman and Tukey (1958) treated many problems that may arise under such circumstances. The most serious pitfalls may be avoided by performing a few very simple preliminary steps.

The main one is band-limiting in the collection of the data. It is important because frequency components outside the range of interest can seriously affect those that are of interest. The band-limiting is easily done with knob settings on many psychophysiological signal amplifiers. A rule of thumb is to set the equipment to severely attenuate variations at frequencies at and above the discrete sampling rate.

Three other simple operations are usually desirable after the digitizing. The first is to subtract the mean of each record from each observation in the record. The second is to remove any linear trend component, by subtracting linearly regressed values from the observations. The third is to taper the record from the middle to both ends. A frequently recommended tapering procedure is to multiply the th observation, in a record containing $n$ equally spaced observations, $i, y .54+.46$ $\cos [\pi(2 t / n-1)]$, for $t=0,1,2, \ldots n-1$. This is a form of the "Hamming data window." For explanations and further details, see Blackman and Tukey (1958) and Kaiser (1975).

\section{ORTHOGONAL POLYNOMIALS}

Kaplan (1983) has illustrated the analogies between polynomial least-squares regression and Fourier analysis. The general methods of analysis of variance are almost identical in the two cases when they are applied in balanced designs. In each case, the procedure can be conceptualized in terms of a multiple-regression equation. In each case, one of the experimental variables is treated as a trend variable, which is quantitative, with points of observation laid out in an ascending sequence along the variable. In the present discussion, the trend variable is time and the points of observation are assumed to be equally spaced. That is frequently the case with polynomial trend analysis, and usually with Fourier analysis. In a simple design, each of several subjects is measured at each of these points in time. Thus, it is a simple treatments-by-subjects or repeatedmeasures design, and necessarily balanced. In the ordinary ANOVA, there is a between-times ( $T$ ) sum of squares, a between-subjects (S) sum of squares, and a $\mathrm{T}$ by $\mathrm{S}$ interaction. The $\mathrm{T}$ mean square is tested against the $\mathrm{T}$ by $\mathrm{S}$ interaction in an $\mathrm{F}$ ratio. There is no test of the $\mathrm{S}$ mean square, which usually is of no interest anyway.

The trend analysis is in addition to the ordinary ANOVA. It is a more microscopic analysis of the pattern of the means at the levels of $T$. The components are additive, and there is a test of the variance contribution of each. In many cases, the objective is to represent the trend of the means in a regression equation that contains only those components that proved significant in the tests of trend, or some subset depending on the outcomes of the tests. In the case of polynomials, the trend components are constant, linear, quadratic, cubic, and so on, and they are of the zeroth, first, second, third degree. and so on. The constant term is of the zeroth degree, and it is simply the grand mean. The use of orthogonal polynomials facilitates the analysis, although ordinary powers of the trend variable could be used in principle, with the calculations done according to the methods of direct stepwise polynomial regression. If the dependent variable is $Y$ and the independent (trend) variable is $\mathrm{X}$, then a polynomial regression equation has the form,

$$
\hat{Y}=c_{0}+c_{1} X+c_{2} X^{2}+\ldots+c_{m} X^{m}
$$

where the regression coefficients, $c_{j}, j=0,1,2, \ldots, m$, are estimated from the data. When orthogonal polynomials are used, the end result is exactly the same, but the regression equation looks different:

$\hat{Y}=$

$d_{0} P_{0}(X)+d_{1} P_{1}(X)+d_{2} P_{2}(X)+\ldots+d_{m} P_{m}(X)$,

were $P_{j}(X)$ is a polynomial in $X$ of Degree $j$. The set of polynomials, $\left[\mathrm{P}_{\mathrm{j}}\right]$, is determined in such a way that their intercorrelations are all zero. The use of $\left[P_{j}\right]$ instead of $[\mathrm{Xj}]$ is simply a technical trick to make it easy to solve for the regression coefficients, and the variance component associated with each, for a given set of data. In Figure 1, one can compare the [Xj] with their orthogonalized counterparts, $\left[P_{j}\right]$. We have $P_{0}=1, P_{1}=X$ (in this case), and for the others, $P_{j}$ is a linear combination of the powers of $X$ up to $j$. The linear combination is determined uniquely, except for a constant multiplying factor, just so that the correlations of $P_{j}$ with those of lower degree are all zero. Each of the $\left[d_{j}\right]$ coefficients in the regression equation (Equation 2) is associated with a single degree of freedom. Here is where the convenience comes in. Since the intercorrelations of the orthogonal polynomials are all zero, each of the $\mathrm{m}$ normal equations determining the regression coefficients is a single equation in a single unknown. In preparation for terminology to be used later, note that the complete set of coefficients, $\left[d_{j}\right]$, forms a nonsingular transform of the original data. If the number of coefficients is the same as the number of original data points, the regression equation will fit the observed data perfectly. Indeed, the transform could then be inverted to recover the original data. To be explicit, the forward transform is $d_{j}=\Sigma Y P_{j} / \Sigma P_{j}^{2}$, and the inverse is simply the regression equation (Equation 2).

Each of the coefficients, $d_{j}$, accounts for its own unique component of the sum of squares of $Y$. A conse- 

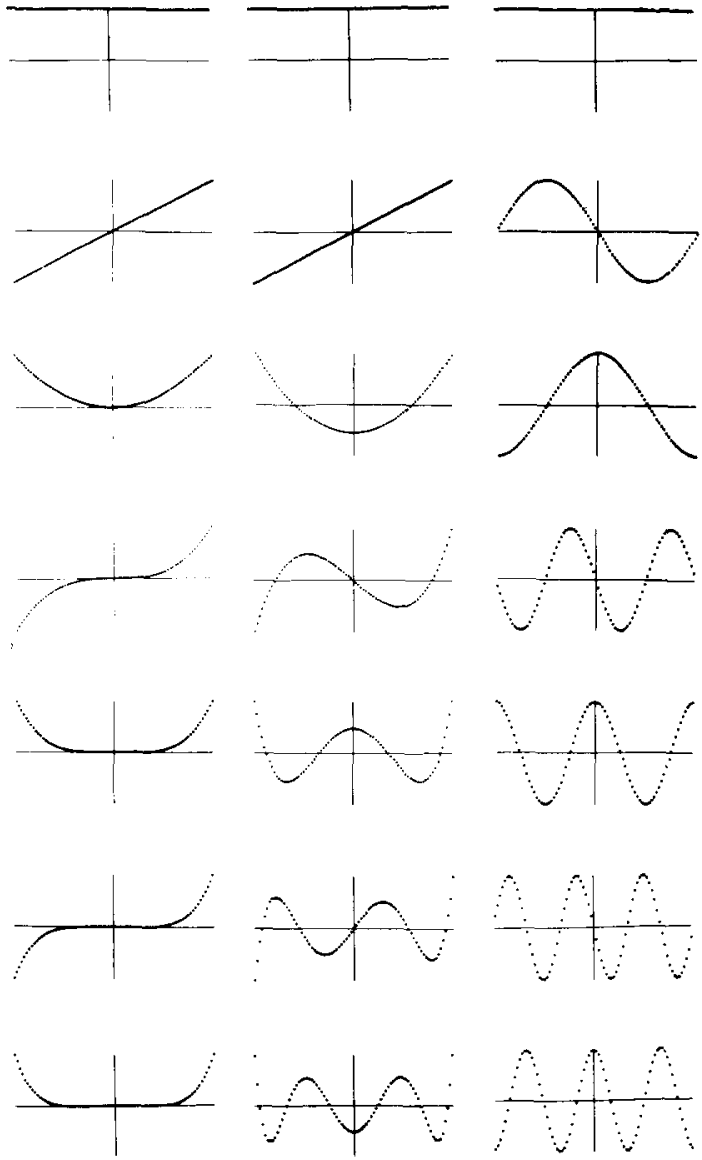

Figure 1. Powers of $\mathbf{X}$ (first column), orthogonal polynomials (second column), and Fourier components (third column).

quence of all this is that the values of $\left[d_{j}\right]$ do not change when the equation is expanded or reduced to include more or fewer terms. Equation 1, on the other hand, does not have this property. Expansion or reduction of the members of $\left[\mathrm{X}^{\mathrm{j}}\right]$ requires a new simultaneous solution for all the coefficients, $\left[c_{j}\right]$. Such properties led Alexander (1946) and Grant (1956), among others, to ANOVA tests for the true regression coefficients of which the $\left[\mathrm{d}_{j}\right]$ are estimates. In the simple $T$ by $S$ design mentioned earlier, the $Y$ variance attributable to $T$ is analyzable into $m$ independent components, where $m+1$ is the number of levels of $T$. Each is associated with 1 degree of freedom. The sum of squares for Component $\mathrm{j}$ is essentially $\mathrm{Nd}_{j}^{2} \Sigma \mathrm{P}_{j}^{2}$, where $\mathrm{N}$ is the number of subjects, and the summation is over the levels of T. Many statistics and design texts outline the computational formulas in detail, but two other logical points deserve mention.

In outlining the use of orthogonal polynomials in a number of designs, Grant (1956) took a conservative view on the choice of error terms. Returning to the simple $\mathrm{T}$ by $\mathrm{S}$ design, Grant's method was essentially as follows. Just as the $T$ sum of squares is analyzable into $\mathrm{m}$ components, each with 1 degree of freedom, the $\mathrm{T}$ by $\mathrm{S}$ sum of squares is analyzable into $\mathrm{m}$ com- ponents, each with $\mathrm{N}-1$ degrees of freedom, where $\mathrm{N}$ is the number of subjects. Thus, for each orthogonal component of $\mathrm{T}$, there is a corresponding orthogonal component of $\mathrm{T}$ by $\mathrm{S}$, and Grant formed $\mathrm{F}$ ratios of such corresponding pairs, with 1 and $\mathrm{N}-1$ degrees of freedom. Others, in order to get more degrees of freedom into the $F$ denominators, resorted to various schemes of pooling noncorresponding $\mathrm{T}$ by $\mathrm{S}$ components. Gaito and Tumer (1963) showed that all such pooling methods are based on implicit assumptions that can be quite implausible when made explicit. Hence, Grant's procedure will be followed entirely in the present discussion.

Note carefully, however, that one can freely pool corresponding numerators and denominators of the $\mathrm{F}$ ratio, if such pooling is done in a parallel way. Gaito and Turner (1963) did not argue against pooling in that way. Thus, for example, the residual of the $T$ main effects after extraction of the linear and quadratic components is testable against the residual of $T$ by $S$ after removing the linear- $\mathrm{xS}$ and the quadratic- $\mathrm{xS}$ components. Tests of such pooled residuals are sometimes used as the criterion for stopping in stepwise analyses. Parallel pooling is also done for other reasons under particular circumstances.

\section{ORTHOGONAL FREQUENCY COMPONENTS}

The researcher who has some familiarity with using orthogonal polynomials to fit curves and test the contributions of the components has a good start toward similar tests with the frequency components. The sine and cosine functions are already orthogonal (Franklin, 1949 , pp. 83-88). With orthogonal polynomials, on the other hand, one must usually refer to published tables or implement a computer program (Emerson, 1982).

If the Fourier components are an alternative to orthogonal polynomials, then what factors affect the choice between them? Some of these factors can be inferred from the shapes of the curves in the sets, as illustrated in Figure 1. Others have to do with the behaviors of the different functions when extended outside the range of the original data. Still others have to do with cases in which the process under study is thought to conform to one theoretical model or another. It should be clear, though, that either would be just as good as the other in satisfying the assumptions for the F tests. Indeed, any other set of orthogonal functions would do just as well as either of these, for that purpose.

Grant (1956) pointed out that orthogonal polynomials are not very good for fitting data that follow asymptotic trends, such as learning curves. The Fourier components, as outlined here, are little better for that purpose. With that application ruled out for both sets, how do they compare in other applications? Figure 1 illustrates one fundamental fact that has relevant implications. The polynomials include a linear component, but the Fourier components do not. If testing for linear 
trend is of importance, then, polynomials would be the more natural choice. Other than that, the mere general shapes of the curves do not suggest anything crucial. For example, either the quadratic polynomial or the first cosine component might serve to test for a general symmetrical U-shaped trend. If that were the only objective, the experimenter might choose according to his subjective notion of which better approximates what he wants to test.

Note that each of the polynomials, except the first, becomes generally steeper and oscillates more rapidly near the ends of the range than near the middle. The increasing steepness continues outside the data limits, but there are no further oscillations. Thus, the curves go rapidly toward infinity, in one direction or the other, as one ventures farther outside the limits. Such extrapolated behavior would rarely be very plausible. If extrapolation along the trend variable is not an objective, then, of course, one does not care how the functions behave outside the limits. The Fourier components behave much differently outside the range for which they are fitted. They simply repeat themselves. They are stationary in the time-series sense, and, more specifically, they are periodic. Any regression equation fitted using the Fourier components is therefore periodic when extrapolated outside the fitting range. That feature has made them the most popular choice for fitting data that are thought to follow some periodic trend. Polynomials have been more popular for most other applications, perhaps because it is frequent that researchers want to test the linear component. It may be rare that a theoretical model predicts exact linearity, but the straight line seems to provide a convenient operational representation of functions that generally increase or decrease across most of the range in question. The test of linear trend compares fairly well with most other operational ways of testing for general monotonic trend (Berenson, 1982).

Most of the applications of the Fourier components have been in cases in which some sort of periodicity is thought to be involved. In those cases, orthogonal polynomials might be a poor choice, if there is the implicit objective of extrapolation making use of the periodicities. The Fourier components are more natural. In this type of application, the components are paired; it is usually convenient to pair them in any case. The paired terms are $\cos (f w t)$ and $\sin (f w t)$. Here, $f$ is frequency, $t$ is time, $w$ is a time-scale factor, $w=2 \pi / n$, and $\mathrm{n}$ is the number of equally spaced time points. It turns out that any linear combination of the two, say, $a \cos (f w t)+b \sin (f w t)$, has exactly the same form as either alone, but with amplitude (vertical magnification) $\sqrt{a^{2}+b^{2}}$ and phase shift (horizontal displacement) $n \times \arctan (b / a) /(2 \pi f)$. Despite these connections, the two members of a pair contribute separate and independent information when included in a least-squares regression equation. An effort will be made to minimize equations and formulas here. However, it seems necessary at least to write the regression equation. It is $\hat{Y}=a_{0}+a_{1} \cos (w t)+a_{2} \cos (2 w t)+\ldots+a_{m} \cos (m w t)$ $+b_{1} \sin (w t)+b_{2} \sin (2 w t)+\ldots+b_{m} \sin (m w t)$. The definitions of $w$ and $t$ are the same as above. It is somewhat important to number the $n$ time points as follows: $\mathrm{t}=0,1,2, \ldots, \mathrm{n}-1$. They could start at 1 instead of 0 and end at $n$ instead of $n-1$, but the interpretation of estimated phases is easier with the present scheme. The regression equation can be written more compactly as $\hat{Y}=a_{0}+\Sigma\left[a_{f} \cos (f w t)+b_{f} \sin (f w t)\right]$, where the summation is from $f=1$ to $f=m$. The regression coefficients, $a_{f}$ and $b_{f}$, are calculated from a data record of $n$ values of $\mathrm{Y}$. They are given by $\mathrm{a}_{0}=\Sigma \mathrm{Y} / \mathrm{n} ; \mathrm{a}_{\mathrm{f}}=2[\Sigma \mathrm{Y} \cos (\mathrm{fwt})] / \mathrm{n}$, $f>0 ; b_{f}=2[\Sigma Y \sin (f w t)] / n, f>0$; where the summations are from $t=0$ to $t=n-1$. Since there cannot be more than $n$ of them, $m$ is limited to $(n-1) / 2$ for $n$ odd. In the case of even $\mathrm{n}$, one more formula is needed: $a_{n / 2}=[\Sigma Y \cos (\pi t)] / n$.

Outlines of proofs of these relations can be found in Franklin (1949, pp. 83-89). In these equations, the pairing of sine and cosine terms is with respect to frequency. The frequency is f cycles/record, but it can be converted to cycles per unit of time by dividing by the duration of the record. The maximum frequency that can be estimated is $(n-1) / 2$ for $n$ odd, or $n / 2$ for $n$ even. This is called the Nyquist frequency, or folding frequency. A vector of all the coefficients for $f=0$ up to the folding frequency, in some prescribed order, is a form of the discrete Fourier transform. Once these coefficients' values have been determined, they can be substituted into the regression equation, and regressed values of $Y$ can be calculated. This process is one way of performing an inversion of the transform, that is, of going from the coefficients (frequency domain) back to the data (time domain). However, the fast Fourier transform algorithm (Emerson, 1980; Liu, 1975) can be used to perform both the forward and inverse transforms faster.

Because the sine and cosine functions form an orthogonal set, the Fourier transform yields a partitioning of the $\mathrm{Y}$ sum of squares into $n$ components, each associated with 1 degree of freedom. Indeed, the sum-ofsquares components are $n a_{0}^{2}$, and $n a_{f}^{2} / 2$, and $n b_{f}^{2} / 2$ for $\mathrm{f}=1,2, \ldots,(\mathrm{n}-1) / 2$, and $\mathrm{na}_{\mathrm{n} / 2}^{2}$ for $\mathrm{n}$ even. The sum of all of these is equal to $\Sigma Y^{2}$, and the sum of all but $\mathrm{na}_{0}^{2}$ is equal to $\Sigma(\mathrm{Y}-\overline{\mathrm{Y}})^{2}$. This fact is a consequence of Parseval's theorem (Carslaw, 1930). If each $Y$ is the mean of, say, $\mathrm{N}$ observations, rather than a single one, then the sums of squares specified above should be multiplied by a weighting factor of $\mathrm{N}$, for purposes of ANOVA. The ANOVA could proceed exactly as in the case of orthogonal polynomials. However, it makes sense to pool the sums of squares for sine and cosine components of the same frequency for $F$ tests with 2 degrees of freedom in the numerator and a proportionate number in the denominator. The pooling for the 
Table 1

Body Temperature as a Function of Time of Day for Two Subjects

\begin{tabular}{llll}
\hline Time & $\mathbf{t}$ & S1 & S2 \\
\hline Midnight & 0 & 1 & 1 \\
4:00 a.m. & 1 & 0 & 0 \\
8:00 a.m. & 2 & 1 & 2 \\
Noon & 3 & 2 & 3 \\
4:00 p.m. & 4 & 4 & 5 \\
8:00 p.m. & 5 & 3 & 2 \\
\hline
\end{tabular}

Table 2

Analysis of Variance With Fourier Analysis of Data in Table 1

\begin{tabular}{lrrrr}
\hline Source & df & \multicolumn{1}{c}{ SS } & \multicolumn{1}{c}{ MS } & \multicolumn{1}{c}{ F } \\
\hline $\mathrm{S}$ & 1 & .333 & .333 & \\
$\mathrm{~T}$ & 5 & 24.000 & 4.800 & $14.4^{*}$ \\
T by S & 5 & 1.667 & .333 & \\
Total & 11 & 26.000 & & \\
$\mathrm{H} 1$ & 2 & 22.167 & 11.083 & $19.0 \dagger$ \\
$\mathrm{H} 2$ & 2 & .500 & .250 & 3.0 \\
$\mathrm{H} 3$ & 1 & 1.333 & 1.333 & 4.0 \\
$\mathrm{~T}$ & 5 & 24.000 & & \\
$\mathrm{H} 1$ by S & 2 & 1.167 & .583 & \\
$\mathrm{H} 2$ by S & 2 & .167 & .083 & \\
H3 by S & 1 & .333 & .333 & \\
T by S & 5 & 1.667 & & \\
\hline
\end{tabular}

$*_{\alpha}<.01 . \quad f_{\alpha}<.05$.

error terms should parallel that for the numerators. By the same logic, one can pool over subsets of frequencies to test hypotheses concerning contributions in composite frequency bands. Again, the error terms should be pooled in a parallel way.

A very simple example will now be presented. Table 1 contains fictitious data for two subjects. The data are body temperatures with the constant of 96 subtracted to reduce the sizes of the numbers. The data are spaced $4 \mathrm{~h}$ apart, for six measurements per subject over a 24-h period. The ordinary repeated-measures ANOVA summary is shown in the top of Table 2. The result of the supplementary analysis for frequency components is given in the bottom of Table 2 . The components are called $\mathrm{H} 1, \mathrm{H} 2$, and $\mathrm{H} 3$, for the first, second, and third harmonics. $\mathrm{H} 3$ is associated with only 1 degree of freedom because $\mathrm{n}$ is even and $\mathrm{f}=3$ is the folding frequency in this case. From the analysis of Table 2, only the contribution of the fundamental (H1) proved significant. Therefore, according to usual practices, the regression equation can be truncated after the fundamental. The result is $\hat{Y}=2-1.0833 \cos (w t)-$ $1.5877 \sin (w t)$. From Table 2, it is seen that this accounts for $100 \times 22.167 / 24=92 \%$ of the variation of the Y means about the grand mean.

\section{HIGHER ORDER DESIGNS}

Very little needs to be said about the technical aspects of compounding the simple repeated-measures design to obtain multiple-way designs with Fourier analysis. The pattern follows that laid out by Grant (1956) for orthogonal polynomials. The main difference, perhaps, is the usual pooling of sine and cosine components of the same frequency, with the pooling done in parallel for numerators and denominators of the $F$ ratios.

Generally, there are two main ways to compound the simple $\mathrm{T}$ by $\mathrm{S}$ design, to obtain higher order balanced designs. One is to replicate it on different groups of subjects, with the groups treated differently in the experiment. The second is to replicate it on the same subjects, with the replications being done under different experimental conditions. It may be compounded more than once in either of the two ways, or in both ways. The general principles are well known.

There are two special options that arise when treating coherent periodic data. One is the use of cycles as an experimental variable of the second kind. In such a case, the length of the elementary record would correspond to one cycle and would be of the fundamental period determined by the period of the driving stimulus. However, data would be collected continually over several repetitions of the cyclic stimulus for each of two or more subjects. The main idea of such use of cycles as an experimental variable would be to test for changes in the form of the response curve from cycle to cycle. The null hypothesis would be perfect periodicity (i.e., no changes). Such an experiment might be quite meaningful even without any Fourier analysis. The cycles main effects (C) would be testable against the $\mathrm{C}$ by $\mathrm{S}$ interaction to see if there were significant changes in the average level of response. The $\mathrm{T}$ by $\mathrm{C}$ interaction would be testable against $\mathrm{T}$ by $\mathrm{C}$ by $\mathrm{S}$ for a general test of curve-form differences in the different cycles. Then why complicate the picture with Fourier analysis?

The reasons have to do with more specific questions that might be of interest concerning the nature of the curve-form changes. A complete example is worked out below, in which a specific question is involved. However, it is connected with another option that is available. It is the analysis of differences in the contribution of a frequency component under different experimental conditions into phase and amplitude differences. Before reviewing the example, it is necessary to discuss the scale on which phases are measures.

Let $\theta^{\prime}=\arctan (b / a)$. If $a$ and $b$ are the cosine and sine coefficients for a given frequency component, then $\theta^{\prime}$ is said to be the phase angle of that component. With the time points numbered as $\mathrm{t}=0,1,2, \ldots, \mathrm{n}-1$, the phase angle gives information about the location of the first peak of the sinusoidal component. However, $\theta^{\prime}$ alone is not sufficient. The signs of $a$ and $b$ must be taken into account too. Computer and calculator arctangent functions usually give $\theta^{\prime}$ in the range from $-\pi / 2$ to $+\pi / 2$. The first step in using $\theta^{\prime}$ is to convert it to $\theta$, which lies in the range from 0 to $2 \pi$, taking into account the signs of $a$ and $b$. This can be done from Table 3. The conversion to a displacement (D) along the time scale then can be done by taking $D=\theta n /(2 \pi f)$. 
Table 3

Conversion of $\theta^{\prime}=\arctan (b / a)$ to $\theta$ in the Interval 0 to $2 \pi$

\begin{tabular}{cll}
\hline $\mathrm{a}$ & $\mathrm{b}$ & \multicolumn{1}{c}{$\theta$} \\
\hline$=0$ & $>0$ & $\pi / 2$ \\
$=0$ & $<0$ & $3 \pi / 2$ \\
$>0$ & $=0$ & 0 \\
$<0$ & $=0$ & $\pi$ \\
$>0$ & $>0$ & $\theta^{\prime}$ \\
$>0$ & $<0$ & $\theta^{\prime}+2 \pi$ \\
$<0$ & $>0$ & $\theta^{\prime}+\pi$ \\
$<0$ & $<0$ & $\theta^{\prime}+\pi$
\end{tabular}

Table 4

Response Rates of Two Subjects at Eight Points in Each of Three Contingency Cycles

\begin{tabular}{|c|c|c|c|c|c|c|}
\hline \multirow[b]{2}{*}{$\mathbf{t}$} & \multicolumn{3}{|c|}{ S1 } & \multicolumn{3}{|c|}{$\mathrm{S} 2$} \\
\hline & C1 & $\mathrm{C} 2$ & $\mathrm{C} 3$ & $\mathrm{C} 1$ & $\mathrm{C} 2$ & C3 \\
\hline 0 & 1 & 1 & 3 & 0 & 2 & 5 \\
\hline 1 & 0 & 1 & 4 & 1 & 1 & 5 \\
\hline 2 & 1 & 3 & 6 & 0 & 2 & 8 \\
\hline 3 & 3 & 3 & 8 & 2 & 5 & 7 \\
\hline 4 & 4 & 4 & 5 & 3 & 5 & 3 \\
\hline 5 & 5 & 3 & 2 & 3 & 4 & 1 \\
\hline 6 & 4 & 1 & 1 & 1 & 1 & 2 \\
\hline 7 & 1 & 3 & 4 & 1 & 3 & 5 \\
\hline
\end{tabular}

Use will be made of these relations in the following example.

A technique sometimes used in operant conditioning experiments is the ABA design. In such an experiment, there is a repetitive turning on and turning off of the response-reinforcement contingency. It is an interesting general design, because it can be varied in many ways. In the present example, there were three complete cycles, and it would be called an $\mathrm{ABABAB}$ design. The durations of the on (A) and off (B) periods were equal, and four measurements of response rate were made within each of these half-cycles, at equally spaced times. Fictitious data are presented in Table 4, for two naive subjects. The main interest in such an experiment would be in whether the response rate varies regularly during the contingency schedule. Also, because the subjects begin with no prior experience in the task, it would be of great interest to see how the response-rate curves change from cycle to cycle. One might conject that the curves would begin with sluggish variation. It would not be surprising if they then began to increase in amplitude and decrease in phase delay on each additional cycle. Many vertebrates exhibit "anticipatory" behavior in situations involving repetitions of the same interstimulus interval. The general ANOVA design is $8 \times$ $3 \times 2$ for $\mathrm{T}$ by $\mathrm{C}$ by $\mathrm{S}$. The $\mathrm{T}$ effects are tested against the $\mathrm{T}$ by $\mathrm{S}$, the $\mathrm{C}$ against the $\mathrm{C}$ by $\mathrm{S}$, and the $\mathrm{T}$ by $\mathrm{C}$ against the $\mathrm{T}$ by $\mathrm{C}$ by $\mathrm{S}$. The first question, logically, involves the $\mathrm{T}$ by $\mathrm{C}$ effects. If they are not significant. then one would naturally look to the $T$ and $C$ effects. In the present case, the $\mathrm{T}$ by $\mathrm{C}$ effects are significant, so the $\mathrm{T}$ and $\mathrm{C}$ main effects become of secondary interest. Also, one naturally becomes curious about how the time trend changes from cycle to cycle. Most of the ANOVA summary is presented in Table 5, including the Fourier trend analysis.

Examining the $\mathrm{T}$ by $\mathrm{C}$ interaction, which is of primary interest, we see that the $\mathrm{H} 1$ by $\mathrm{C}$ component accounts for most of it and is significant at the .05 level. Therefore, the fundamental is plotted in the top graph of Figure 2 for each of the three cycles. There are discontinuities from one cycle to the next because of the method of analysis chosen for this case. Although these are unaesthetic and unrealistic, they are minor consequences of the departures from periodicity that are tested by the $\mathrm{T}$ by $\mathrm{C}$ interaction. In the bottom graph in Figure 2, the $\mathrm{H} 2$ by $\mathrm{C}$ component of $\mathrm{T}$ by $\mathrm{C}$ has been included with the $\mathrm{Hl}$ by $\mathrm{C}$ component. This violates the law of parsimony, since $\mathrm{H} 2$ by $\mathrm{C}$ was not significant, but it is of interest from a methodological viewpoint to see how an added term improves the apparent fit.

Bliss (1958), in analyses of meteorological data, divided the interaction sum of squares for a frequency component into two parts that he attributed to phase and amplitude differences. He constructed error terms for testing them separately. Phases and amplitudes are nonlinear functions of the Fourier coefficients, so their differences are not exactly normally distributed. It was Bliss' policy to test separately for amplitude and phase differences only after the overall test of the interaction of the frequency component proved significant. Presumably, this is because the approximation to normality

Table 5

Partial Summary of Analysis of Variance With Fourier Analysis of Data in Table 4

\begin{tabular}{|c|c|c|c|}
\hline Source & $d f$ & SS & $\mathrm{F}$ \\
\hline H1 & 2 & 31.675 & 10.3 \\
\hline $\mathrm{H} 2$ & 2 & 10.875 & $52.2^{*}$ \\
\hline $\mathrm{H} 3$ & 2 & 1.741 & $182.0 \dagger$ \\
\hline H4 & 1 & 1.688 & 9.0 \\
\hline $\mathrm{T}$ & 7 & 45.979 & $13.2 \dagger$ \\
\hline $\mathrm{H} 1$ by $\mathrm{S}$ & 2 & 3.074 & \\
\hline $\mathrm{H} 2$ by $\mathrm{S}$ & 2 & .208 & \\
\hline $\mathrm{H} 3$ by $\mathrm{S}$ & 2 & .010 & \\
\hline $\mathrm{H} 4$ by $\mathrm{S}$ & 1 & .188 & \\
\hline $\mathrm{T}$ by $\mathrm{S}$ & 7 & 3.479 & \\
\hline $\mathrm{H} 1$ by $\mathrm{C}$ & 4 & 58.053 & $9.8^{*}$ \\
\hline $\mathrm{H} 2$ by $\mathrm{C}$ & 4 & 14.500 & 5.4 \\
\hline $\mathrm{H} 3$ by $\mathrm{C}$ & 4 & 2.781 & 1.2 \\
\hline $\mathrm{H} 4$ by $\mathrm{C}$ & 2 & .125 & .1 \\
\hline $\mathrm{T}$ by $\mathrm{C}$ & 14 & 75.458 & $6.1 \dagger$ \\
\hline $\mathrm{Hl}$ by $\mathrm{C}$ by $\mathrm{S}$ & 4 & 5.910 & \\
\hline $\mathrm{H} 2$ by $\mathrm{C}$ by $\mathrm{S}$ & 4 & 2.667 & \\
\hline $\mathrm{H} 3$ by $\mathrm{C}$ by $\mathrm{S}$ & 4 & 2.257 & \\
\hline $\mathrm{H} 4$ by $\mathrm{C}$ by $\mathrm{S}$ & 2 & 1.625 & \\
\hline T by C by $S$ & 14 & 12.459 & \\
\hline
\end{tabular}

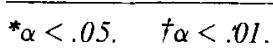




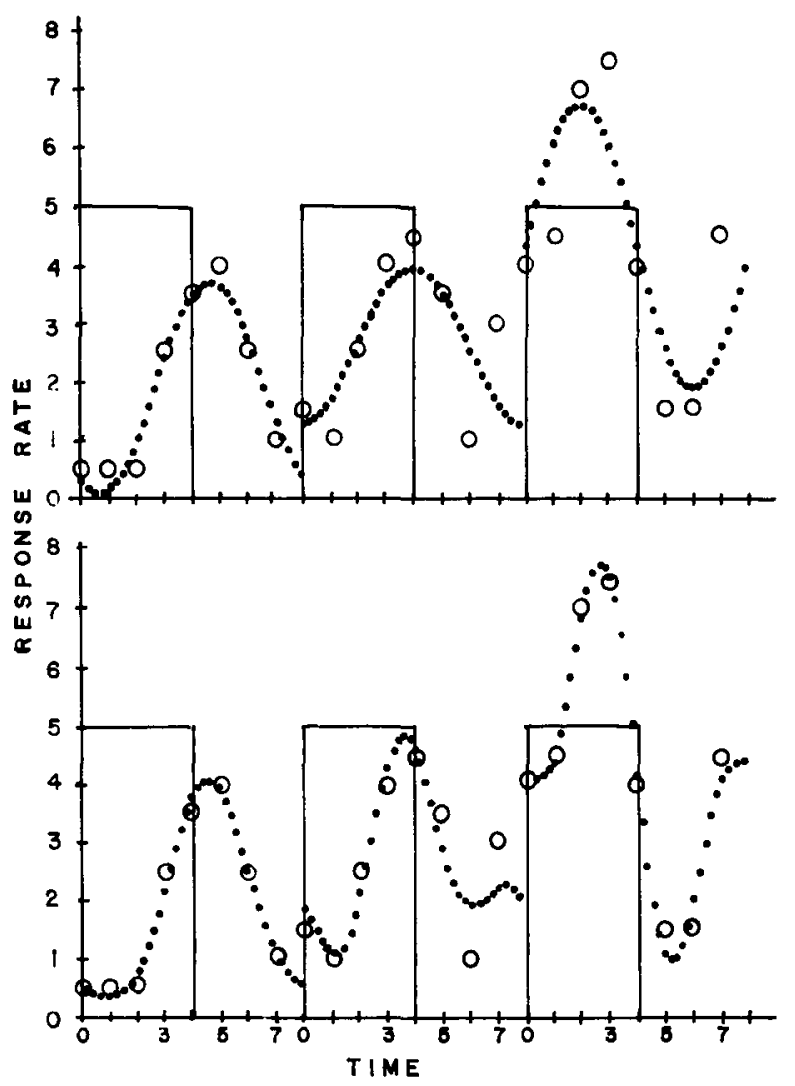

Figure 2. The fundamental frequency component (top graph) and the fundamental combined with the second harmonic (bottom graph), fitted to the means for the two subjects for each cycle. Data are from Table 4.

of the differences is good only when amplitudes are large and phase differences are small within conditions.

In the present example, it is of interest to make separate tests of the amplitude and phase differences of the fundamental frequency component at the three levels of $\mathrm{C}$. The question is whether the significant $\mathrm{H} 1$ by $\mathrm{C}$ interaction is due mainly to amplitude differences or to phase differences, or whether both contribute about equally. The simplest procedure, although it does not exactly divide the $\mathrm{H} 1$ by $\mathrm{C}$ sum of squares into two additive components, is started by obtaining the amplitude and phase for each $\mathrm{C}$ by $\mathrm{S}$ combination. Then a separate analysis can be done on each in a $\mathrm{C}$ by $\mathrm{S}$ design in which $\mathrm{C}$ is tested against the $\mathrm{C}$ by $\mathrm{S}$ interaction. This was done, and it turned out that the amplitude differences were not significant at the .05 level. The phase differences were, and they approached the .01 level $[F(2,2)=94.2]$. This indicates that the differences between the three amplitudes, 1.84, 1.37, and 2.44 , contributed less than the differences between the three phase displacements, $4.61,3.90$, and 2.00 . These displacements give the time-scale locations of the first maxima of the three curves, as they have been computed by the method associated with Table 3 .
Their decreasing trend is clearly consistent with the prior conjecture of the growth of anticipation with increasing experience in the repetitive time schedule.

\section{PLANNING A MORE COMPLEX CASE}

The preceding example is on the fringe of those situations in which Fourier analysis is ordinarily performed. It illustrates the makeshift use of a tool when no better tool seems available. For example, it is not easy to see any better way that orthogonal polynomials might have been used to test for the systematic time shifts. Both of the preceding examples are probably simpler than any situation of interest in actual research. In both cases, the conditions did not require the kind of precautions that are often necessary. Therefore, let us consider, without a numerical example, a situation that might be more typical. The type of planning required is essentially the same as that outlined by Blackman and Tukey (1958). However, their general practice was to Fourier transform autocovariances rather than the time series directly. The Fourier transform of the autocovariances yields the power, $a^{2}+b^{2}$, for each frequency instead of the pair of quantities, $a$ and $b$. For the coherent analysis, the separate estimates of $a$ and $b$ are needed. Scattered in the pages of Blackman and Tukey (1958), one finds brief discussions of the alternate method, the one needed here. In particular, they discuss the tradeoff between frequency resolution and stability of estimates (Blackman \& Tukey, 1958, p. 95). That tradeoff is important in the following example of planning.

Assume that scalp voltages are to be recorded while a sinusoidally modulated visual stimulus is presented. Assume that the recordings are from a number of electrode sites (say, five) in an array oriented along a path thought to be the direction of propagation of cortical waves set up by the periodic stimulus. Assume that it is feasible to maintain stimulating and other conditions constant only for a short time, so that only about $5 \mathrm{sec}$ of artifact-free record can be obtained at a stretch. Assume that the frequency of the sinusoidal photic stimulus is 9 cps.

Since scalp potentials are typically very noisy, we must take the precautions mentioned earlier. The first question concerns the setting of the low-pass characteristic of the filters of the amplifiers. If it is of interest to test for nonlinearity of the stimulus-response relation, the upper band limit should probably be set so that at least the second and third harmonics are passed. The third is at $27 \mathrm{cps}$, so if there is a knob setting for $50 \mathrm{cps}$ on the amplifiers for the high band limit, it might be appropriate. Assuming that the gain is $30 \mathrm{~dB}$ down at $50 \mathrm{cps}$, we might want to sample the amplifier outputs at $100 \mathrm{cps}$. This gives a folding frequency of $50 \mathrm{cps}$ and should satisfy the rule of thumb of severe attenuation at and above the frequency of sampling. 
This will be fast sampling. An analog-to-digital (A/D) conversion will have to be done on each of the five amplifier outputs within each $.01 \cdot \mathrm{sec}$ period. Each A/D conversion and storage operation must therefore be done in about $2 \mathrm{msec}$. This is probably possible with available equipment and a FORTRAN control program. The computer should control the visual stimulus too, though, to maintain the synchronization. Now we must ask how much data will result from this process in about $5 \mathrm{sec}$. Let us make that $5.12 \mathrm{sec}$. Then $\mathrm{n}=512$, which is an integral power of 2, which is required by some fast Fourier transform programs. At one word every $2 \mathrm{msec}$, this will amount to 2,560 words. If the computer has $24 \mathrm{~K}$ words of usable memory, we can very probably store the five 5.12-sec records in memory in integer form with no necessary disk or tape operations until the records are complete.

The next question concerns the digital preprocessing before the Fourier transform is applied. This depends partly on whether or not the amplifiers also have filtering to reject low frequencies. If they can be set to reject all below $2 \mathrm{cps}$, for example, that will essentially remove constant and linear trends. That leaves the data window. The frequency resolution is approximately the reciprocal of the length of the data window. To follow a simple practice, the window will be used on each full 5.12-sec record of 512 data points. Then the frequency resolution is about $.2 \mathrm{cps}$. This is probably finer than desired in view of the nature of scalp potentials.

The elementary frequency estimates will be spaced $1 / 5.12 \mathrm{cps}$ apart with $\mathrm{f} 1=1 / 5.12, \mathrm{f} 2=2 / 5.12 \mathrm{cps}$, and so on, and there will be 256 of them, the highest being at $50 \mathrm{cps}$. Although this seems too fine for the present purposes, it was dictated by the desire to estimate frequency components up to $27 \mathrm{cps}$, by the availability of the 50-cps band-limit knob, and by the rule of sampling at a rate at or above the frequency cutoff. Each of the 256 estimates will be more variable than necessary. There are several things that can be done to deal with this problem.

The most straightforward solution is simply to combine frequency estimates into subbands. We would like to center subbands at 9,18 , and $27 \mathrm{cps}$. These fall at the estimates indexed 46,92 , and 138 . Within each of the subbands, let us include in the sum the two adjacent elements in each direction. Thus, the estimates indexed $44,45,46,47$, and 48 will be combined, for $9 \mathrm{cps}$, and similarly for 18 and $27 \mathrm{cps}$. The subband estimates, say, B9, B18, and B27, are now about $1 \mathrm{cps}$ in width and will be more reliable than the elementary estimates. The summing within each subband will be done on the sine and cosine components separately.

To proceed with the planning, there will be an $F$ test with 2 and $2(\mathrm{~N}-1)$ degrees of freedom for the overall contribution at each of the three subbands, B9, B18, $B 27$, where $\mathrm{N}$ is the number of subjects or the number of replications on a single subject. The null hypothesis in each case is that the true subband contribution is zero. In addition, and of more interest, is the interaction of each subband contribution with the five electrode sites (E). The B9 by $\mathrm{E}$ component would be tested against $\mathrm{B} 9$ by $\mathrm{E}$ by $\mathrm{S}$, for example, with 8 and $8(\mathrm{~N}-1)$ degrees of freedom. Let us say that the B 9 by $\mathrm{E}$ test proves significant, but the $\mathrm{B} 18$ by $\mathrm{E}$ and $B 27$ by $E$ do not. That is perhaps what one might hope. Then the test of the phase-difference component of B9 by $\mathbf{E}$ would be justified, according to Bliss' (1958) policy. This test could be made in essentially the same way as the phase-difference test was made in the second example. If it were to prove significant, one could then get into the intriguing logical problems of inferring the direction and speed of the cortical waves set up by the periodic stimulus from the average time differences among electrode sites.

This planning obviously neglects many control problems such as the matching of the five amplifiers and electrode contact resistances. Poor control of such factors could yield spurious phase and amplitude differences. This planning example goes a bit beyond the purely empirical cut-and-dry method in which many pilot studies might be required. Still, the scheme might fail because the subbands are too broad or too narrow, or for many other reasons. If something goes wrong, plots of the complete spectra, in some form, might provide a clue to the difficulty

The case of noncoherent records has not been treated in this paper, for several reasons. First, it is harder to find simple examples in which the orthogonal-polynomial analogies are meaningful. Second, one has to combine replicates nonlinearly and deal with nonnormal estimates at an earlier stage. Third, such a treatment would likely turn out to be very similar to that of Blackman and Tukey (1958), with their suggested ANOVA on the logarithms of the power estimates.

\section{REFERENCES}

Alexanden, H. W. A general test for trend. Psychological Bulletin, 1946, 43, 533-557.

Berenson, M. L. A comparison of several $k$ sample tests for ordered alternatives in completely randomized designs. Psychometrika, 1982, 47, 265-280.

Blackman, R. B., \& Tukey, J. W. The measurement of power spectra. New York: Dover, 1958.

BuIss, C. I. Periodic regression in biology and climatology. Bulletin 615. New Haven: Connecticut Agricultural Experiment Station, 1958.

Carslaw, H. S. Introduction to the theory of Fourier series and integrals (3rd ed.). New York: Dover, 1930.

Emerson, P. L. Fast Fourier transform fundamentals and applications. Creative Computing, July 1980, pp. 58-63.

Emerson, P. L. Orthogonal polynomial programs: Good vs. better. Behavior Research Methods \& Instrumentation, 1982, $14,489-490$

Franklin, P. An introduction to Fourier methods and the Laplace transformation. New York: Dover, 1949.

Gaito, J., \& Turnen, E. D. Error terms in trend analysis. Psychological Bulletin, 1963, 60, 464-474.

Grant, D. A. Analysis of variance tests in the analysis and comparison of curves. Psychological Bulletin, 1956, 53, 141-154. 
KaISER, J. F. Digital filters. In B. Liu (Ed.), Digital filters and the fast Fourier transform. New York: Halstead, 1975.

KAPLAN, H. L. Correlations, contrasts, and components: Fourier analysis in a more familiar terminology. Behavior Research Methods \& Instrumentation, 1983, 15, 228-241.

LIU, B. (Ed.) Digital filters and the fast Fourier transform. New York: Halstead, 1975.

Porges, S. W., Bohrer, R. E., Cheung, M. N., Drasgow, F., McCABE, P. M., \& Gideon, K. New time-series statistic for detecting rhythmic co-occurrence in the frequency domain. The weighted coherence and its applications to psychophysiological research. Psychological Bulletin, 1980, 88, 580-587.

\section{NOTE}

1. The topic was the Pythagorean theorem, not Fourier analysis. The two are closely related, though. 